\title{
A Built-In-Test Circuit for Functional Verification \& PVT Variations Monitoring of CMOS RF Circuits
}

\author{
Guoyan Zhang, Magdalena Sánchez Mora, Ronan Farrell \\ Institute of Microelectronics and Wireless Systems \\ National University of Ireland, Maynooth \\ Email: gzhang@eeng.nuim.ie
}

\begin{abstract}
Built-In-Test (BIT) for Radio Frequency (RF) integrated circuits can reduce the testing cost, especially with the increase of integration level and operating frequency. A fully integrated CMOS BIT detection circuit is presented in this work. This BIT detection circuit is rectifier-based and low threshold voltage diode-connected MOS transistor with substrate positively-biased is used to improve the detecting sensitivity. As an example, a 2.4GHz LNA is used, the high frequency small signal gain is extracted and the gain fluctuation due to Process, supply Voltage and Temperature (PVT) variations is also investigated. The simulation results show that this BIT detection circuit can realize on-chip functional verification of $\mathrm{RF}$ circuits and also monitor the influence of PVT variations on the performance of the circuit without affecting the high frequency performance of the measured RF circuits.
\end{abstract}

\section{INTRODUCTION}

With the scaling down of CMOS process technology, bulk silicon CMOS process technology has been increasingly used for Radio Frequency (RF) integrated circuits in recent years. The main benefits from the scaling down of CMOS devices are the increase in transit frequency $\left(\mathrm{f}_{\mathrm{t}}\right.$ ) and greater integration, which make RF integrated circuits feasible based on bulk silicon CMOS process. However, the continual scaling down of feature size causes increased difficulty in testing RF circuits with high operating frequencies. There are two main problems for the testing of RF circuits; relaying the multi-gigahertz RF signal to the external tester without affecting the performance of RF circuit under test; and prohibitively expensive RF production testers. Reliable high frequency testing has become a significant restrictive and influential factor for the time-to-market of novel wireless products [1-3]. Therefore, in order to reduce the time and cost, it is important to find an easier way to do the functional verification of RF circuits.

Built-in-Test (BIT) detection circuits provide a flexible way to test the output signal of RF IC by including circuits on-chip that allow the high frequency output signal to be tested by a lower frequency or DC external tester [4-7]. BIT circuits can transform the RF output signal into DC signal, and this approach has been used for functional verification and production real-time monitoring. So, testing of RF integrated circuits with DC or low frequency testing equipments becomes easy and the testing cost is reduced to a large extent, especially with the increase of integration level and operating frequency. Since the last two decades BIT or BIST (Built-In-Self-Test) strategies have been a common practice for the testing of digital and mixed-signal systems [8-10]. However, when considering RF circuits, there are some problems related to RF BIST testing, the need for powerful ADC and DACs, cost in additional chip area, power, etc. In order to reduce the high frequency testing cost and monitoring the performance of the designed RF circuits during product evaluation, some works have been done to develop efficient BIT testing techniques for the RF circuits [11-17]. Recent efforts for RF circuit testing have focused on the design of on-chip embedded detectors, or sensors, where the output signals can be tested easily [13, 16-17], and on the methodologies and algorithms for automated test design $[15,16]$. However, it is not an easy task to design an 
integrated BIT detection circuit. Generally, the BIT detection circuit should not influence the performance of the tested RF circuit, should not consume too much chip area or power. At the same time, the BIT detection circuit should have high sensitivity and can detect weak RF signals. Most importantly, the output results of the BIT detection circuit should reflect necessary information such as the gain of the tested RF circuits. In addition, BIT circuit should be robust to process, supply voltage and temperature (PVT) variations which affect the performance of RF IC to a large extent.

Integrated circuit (IC) manufacturing variations can be placed in two categories: random variations; and systematic variations. Random variations are inherent fluctuations in process parameters, such as those due to random dopant fluctuations from die-to-die, wafer-to-wafer and lot-to-lot. On the other hand, systematic variations depend on the layout pattern and are therefore predictable [18-19]. Process variation can greatly influence the performance of an integrated circuit. In addition, the fluctuation of supply voltage and temperature also influence the performance of the circuit. Thus, performance fluctuation due to PVT variation must be evaluated easily from the output of BIT detection circuit.

As the BIT circuit is optimized for the circuit under test (CUT), and as the set-up and performance requirements for the external tester are drastically reduced, the testing time can also decrease. Figure 1 shows the test circuit schematic with on-chip BIT detection circuit. There are several points in RF circuits and systems can be accessed to evaluate the high frequency performance. In the analyses, the stimulus is provided by external signal source, but the response of different circuits can be found through the BIT detection circuit.

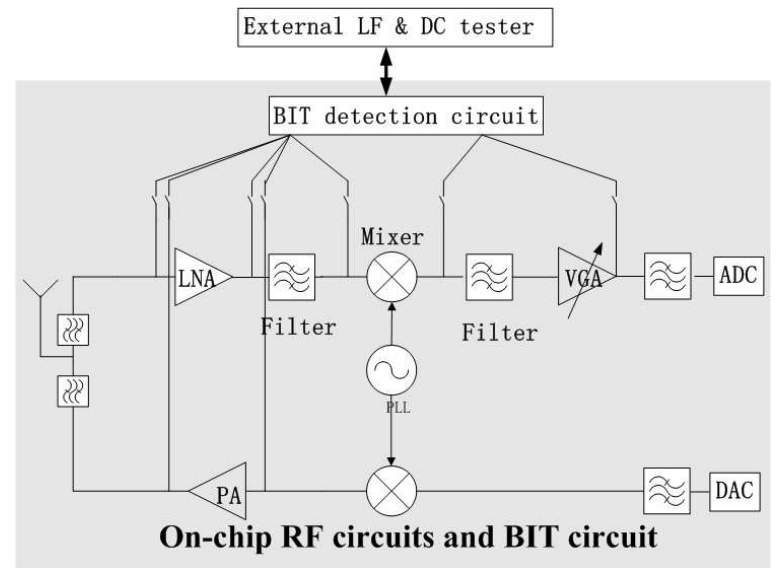

Figure 1: the test of circuit schematic with BIT and tested RF circuits
In this paper, a BIT detection circuit for the testing of RF integrated circuits is proposed. As an example, a 2.4 $\mathrm{GHz}$ LNA is designed and its high frequency small signal gain has been extracted and verified by using a novel BIT detection circuit. At the same time, process, supply voltage and temperature variations are considered during the analysis, and the gain difference of the LNA circuit due to PVT variations is investigated by using the results extracted from the output of BIT circuit. The BIT detection circuit used is rectifier-based, utilizing substrate positively biased low threshold voltage MOS transistors to act as rectifying diodes. The high frequency small signal gain of the LNA circuit can be obtained without influencing the performance of the tested RF circuit. Due to the adoption of substrate-positively-biased scheme, this BIT detection circuit has higher sensitivity to detect weaker input RF signal. In section II, the BIT detection circuit is presented and the performance of the transformation from RF signal to DC signal is verified. The $2.4 \mathrm{GHz}$ LNA circuit is given in section III with details of its high frequency small signal performance provided. Section IV includes the functional verification and the analyses of the monitoring of PVT variations by using the result from BIT detection circuit. The paper concludes with some results and conclusions about the validity of using this novel BIT circuit for high frequency circuit testing..

\section{PROPOSED BIT CIRCUIT}

Figure 2 illustrates the BIT detection circuit, which is a charge pump rectifier configuration proposed for high voltage generation [20]. By using the diode-connected MOS transistors and capacitors, this circuit can

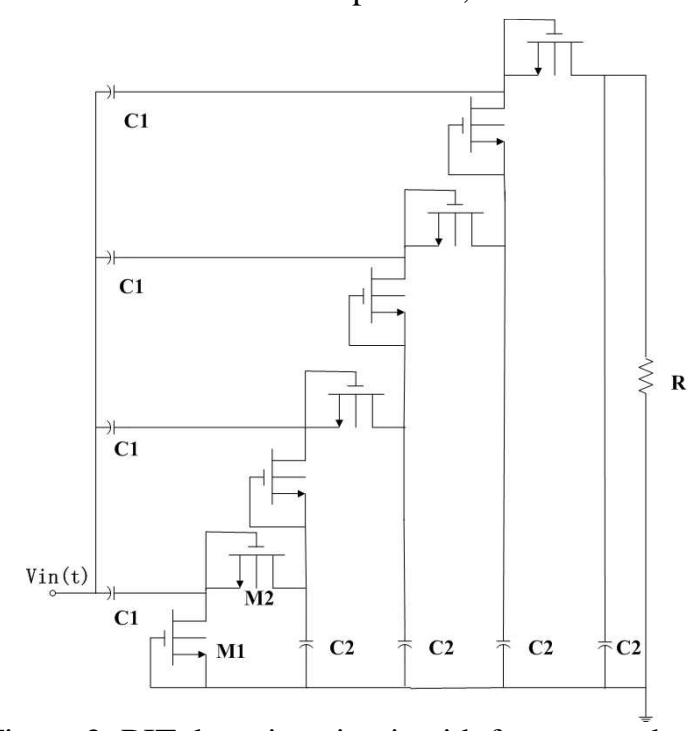

Figure 2: BIT detection circuit with four-stage charge pump rectifier configuration 
transform the input high frequency signal to a corresponding DC signal. For one stage operation, $\mathrm{C}_{1}$ and $\mathrm{M}_{1}$ will shift the input voltage up at point $A$ and $C_{2}$ and $\mathrm{M}_{2}$ will rectify the voltage at $\mathrm{A}$. By using this method, the rectifier delivers a constant output current and a constant output voltage. Figure 3 is a graph showing the relationship of rectifier output voltage to the RF input signal amplitude (a1). The input voltage amplitude sweeps from $0.1 \mathrm{~V}$ to $1 \mathrm{~V}$ in $0.2 \mathrm{~V}$ steps, and the operating frequency is $2.4 \mathrm{GHz}$. From the result it can be seen that the input high frequency signal can be transformed into DC value and the output voltage varies with the input signal amplitude. As there is no biasing voltage needed, this BIT circuit is a passive one, and a separate supply pad during fabrication for the separate control of this detection circuit is not needed. The parameters of the components in the circuits shown in Figure 2 are $\mathrm{C}_{1}=100 \mathrm{fF}$ ), $\mathrm{C}_{2}=700 \mathrm{fF}$, and the channel length and width are $0.3 \mathrm{um}$ and $0.35 \mathrm{um}$ respectively. The capacitance of $\mathrm{C}_{2}$ is a little larger, but it can be used as on-chip decoupling capacitor to filter the noise coming from the supply. The circuit parameters can be optimized to give the most optimal performance according to the requirement of the circuit specifications.

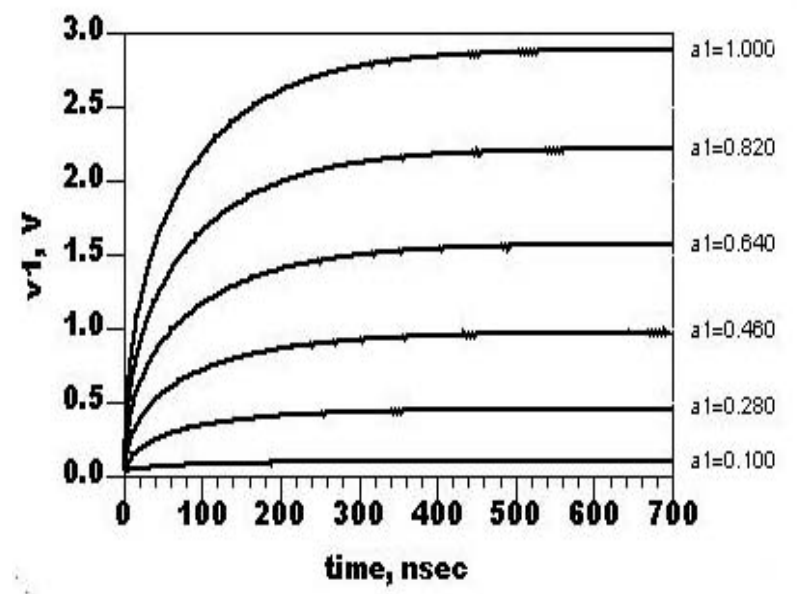

Figure 3: DC output value of BIT detection circuit in figure 2 at different input signal amplitude

In order to detect the weak RF input signal, a lower turn-on-voltage diode is required. To achieve this, the threshold voltage of diode-connected MOS transistor should be low. For our example, a UMC 0.18um low-threshold-voltage MOS transistor was selected for use in the rectifier circuit. By using this low threshold voltage MOS transistor the ability of this circuit to detect weak input signal can be improved. At the same time, a substrate-positively-biased scheme with $0.6 \mathrm{~V}$ is also adopted to further reduce the turn-on voltage of diode-connected MOS transistor and to improve the detecting sensitivity. The validity of using positively-biased substrate for the improvement of detecting sensitivity can be seen in Figure 6 .

\section{LNA CIRCUIT EXAMPLES}

A $2.4 \mathrm{GHz}$ low noise amplifier (LNA) (in Figure 4) has been designed and was used to investigate the validity of this BIT detection circuit for the RF circuit testing, and for the examination of the influence of PVT variations on performance. This LNA includes two stages with a fully on-chip input matching network. The UMC 0.18um mixed signal design kits was used, and the high frequency small signal performance of the LNA is summarized in table I.

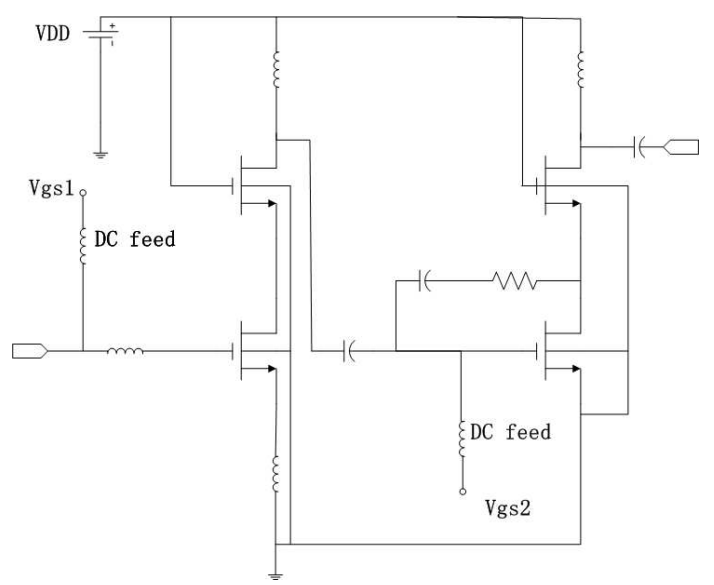

Figure 4: $2.4 \mathrm{GHz}$ LNA circuit

Table I the HF performance of $2.4 \mathrm{GHz}$ stand-alone

\begin{tabular}{|c|c|c|c|c|}
\multicolumn{5}{c}{ low noise amplifier } \\
\hline $\begin{array}{c}\text { Frequency } \\
(\mathrm{GHz})\end{array}$ & $\begin{array}{c}\text { Gain } \\
(\mathrm{dB})\end{array}$ & $\begin{array}{c}\text { NF } \\
(\mathrm{dB})\end{array}$ & $\begin{array}{c}\text { S11 } \\
(\mathrm{dB})\end{array}$ & $\begin{array}{c}\text { IP1dB } \\
(\mathrm{dBm})\end{array}$ \\
\hline 2.4 & 18.64 & 2.16 & -9 & -10 \\
\hline
\end{tabular}

\section{RESULTS}

For the results in this section, the Agilent ADS simulator was used for the simulations, and to verify the validity of the transformation from RF signal to DC signal.

\section{a) Functional Verification}

In this section, this BIT detection circuit for RF testing and for the monitoring of PVT variations of CMOS RF IC is verified by attaching the BIT detection circuit to the tested LNA circuit. This is called combined circuit in the following analyses. This combined circuit includes the LNA circuit in figure 4 and BIT detection circuit in 
figure 2 which connect these two circuits in the way shown in figure 1 . The high frequency small signal gain of LNA circuit can be obtained by extracting from the DC output signal of BIT detection circuit without using a high frequency external tester.

During the analysis, the input signal amplitude sweeps from $-40 \mathrm{dBm}$ to $0 \mathrm{dBm}$ in steps of $1 \mathrm{~dB}$. Figure 5 shows the output time domain signal of the combined circuit. It can be seen that this circuit can realize the transformation from RF output signal to DC signal with different input RF signal amplitude of low noise amplifier.

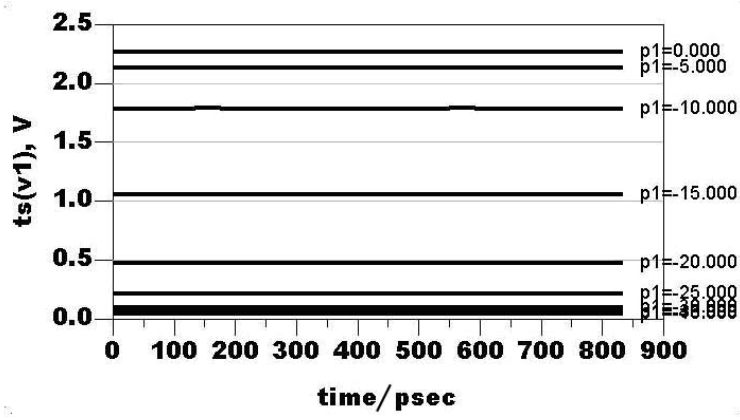

Figure 5: the output transient signal of the combined circuit

Figure 6 evaluates the effectiveness of the positive substrate bias (PSB) of MOS transistors used in BIT detection circuit. With substrate-positively-biased diode-connected MOS transistors in the BIT detection circuit, increased sensitivity can be obtained, for example, for $-40 \mathrm{dBm}$ input power, the output voltage of the combined circuit is $42 \mathrm{mV}$ (marker $\mathrm{m} 1$ ). The output is $6 \mathrm{mV}$ (marker $\mathrm{m} 2$ ) when the substrate-positively-biased scheme is not used. This indicates the significant improvement in sensitivity that can be achieved by adopting substrate positively biased scheme.

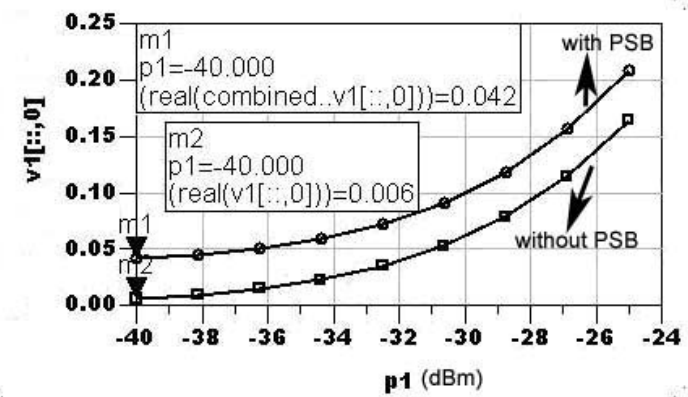

Figure 6: the sensitivity comparison with and without substrate-positively-biased MOS transistors

The high frequency small signal gain of LNA is extracted by using BIT detection circuit. Figure 7 gives the extracted high frequency small signal gain of the LNA, where the BIT detection circuit is attached to the input and output of tested LNA circuit respectively. The curve with marker $\mathrm{m} 2$ is the output of BIT detection circuit when attached to the input of LNA and the curve with marker $\mathrm{m} 1$ is the output of the BIT detection circuit when attached to the output of LNA. The input amplitude $\mathrm{p} 1$ is swept from $-40 \mathrm{dBm}$ to $0 \mathrm{dBm}$ in steps of $0.1 \mathrm{dBm}$. The gain of LNA can be measured as the distance in $\mathrm{dB}$ between marker $\mathrm{m} 1$ and $\mathrm{m} 2$ when two curves enter into linear region. It can be found that the gain is $18.4 \mathrm{~dB}$, and the extracted gain has much difference with the high frequency gain of LNA. From this figure, it can be concluded that this BIT circuit can do functional verification for the CMOS RF circuits.

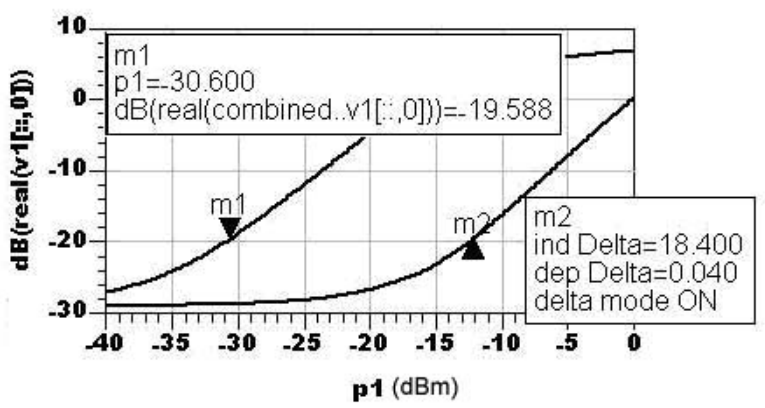

Figure 7: the high frequency small signal gain extracted from the output of BIT circuit attached both at the input and output of LNA

Table II summarizes this $2.4 \mathrm{GHz}$ LNA output high frequency performance with and without BIT circuit attached. It can be seen by comparison of the data shown in table I that there is little influence of the high frequency performance of the LNA circuit due to the attached BIT detection circuit. This is critical for any BIT circuit for RF systems.

Table II the performance of $2.4 \mathrm{GHz}$ low noise amplifier with and without the BIT detection circuit attached

\begin{tabular}{|c|c|c|c|c|}
\hline Circuit & $\begin{array}{c}\text { Gain } \\
(\mathbf{d B})\end{array}$ & $\begin{array}{c}\text { NF } \\
(\mathbf{d B})\end{array}$ & $\begin{array}{c}\text { S11 } \\
(\mathbf{d B})\end{array}$ & $\begin{array}{c}\text { IP1 } \\
(\mathbf{d B m})\end{array}$ \\
\hline with BIT & 18.62 & 2.16 & -9 & -10 \\
\hline without & 18.64 & 2.16 & -9 & -10 \\
\hline
\end{tabular}

\section{b) PVT Variations Monitoring}

With this BIT circuit the high frequency small signal gain of LNA can be obtained without using high frequency tester. However, the influence of process, supply voltage and temperature variations on the performance of CMOS RF circuits needs to be fully evaluated. Because this BIT detection circuit is rectifier based, most of circuit components work at a lower frequency than the input signal frequency, except for the first transistor and capacitor. So, the performance of this BIT detection circuit is not going to be influenced so 
severely as the RF circuit by process or voltage variations which impact on device speed. This BIT detection circuit can be used to evaluate the influence of PVT variations on the performance of RF circuit. During the analysis, $\pm 20 \%$ and $\pm 10 \%$ percentage variations are used for all the parameters in the combined circuit, the supply voltage changes from $1.6 \mathrm{~V}$ to $2.0 \mathrm{~V}$ and operating temperature varies from $-35^{\mathrm{O}}$ to $+80^{\mathrm{O}}$. The difference in small signal gain of the stand-alone LNA and that extracted from the output of the combined circuit was compared under different PVT variations. For process variation, two extreme process variations are included, namely all parameters in the combined circuit vary from unchanged to the plus or minus maximum percentage variation.

Figure 8 shows the gain difference due to the process, supply voltage and temperature variations. Shown in the figure are the gain differences extracted from the output of BIT circuit, and that obtained from the stand alone LNA. From the results obtained, and those shown in Figure 8, this BIT scheme is able to accurately measure the gain variations, to within $0.5 \mathrm{~dB}$ of the LNA for significant variations in process and voltage. For significant temperature variations, the BIT circuit is not as accurate as each transistor in the BIT circuit is wull experience small variations in threshold voltage due to temperature changes. However even over a $50^{\circ}$ variation, the error is only $1.5 \mathrm{~dB}$. Thus it can be said, PVT variations can be detected and monitored through this on-chip circuit.

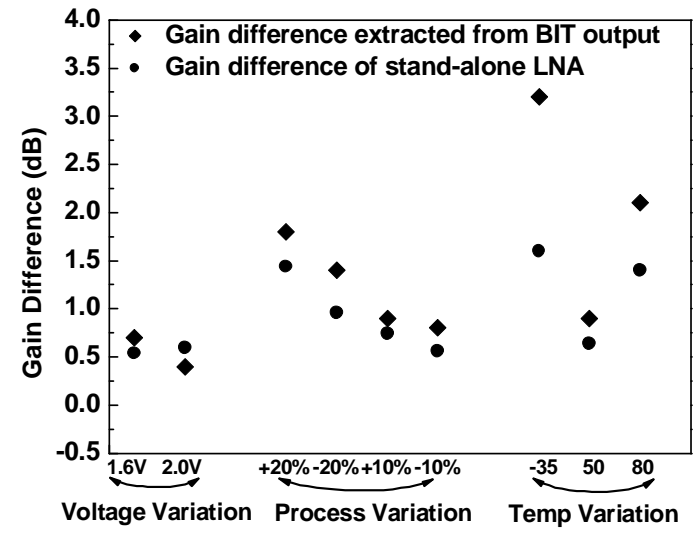

Figure 8: the comparison of gain difference due to PVT variations

\section{CONCLUSIONS}

In this work, a CMOS BIT circuit is presented for RF IC testing. This on-chip circuit can measure the power of high frequency signals allowing accurate gain measurements to be made. The validity of this BIT detection circuit for the functional verification of RF circuits and the evaluation of the influence of the PVT variations on the performance of RF circuits have been tested by using $2.4 \mathrm{GHz}$ LNA circuit. This BIT detection circuit is rectifier-based and utilizes low threshold voltage MOS transistors with -positively-biased substrates to act as diodes to enhance sensitivity. For functional verification high frequency small signal gain can be extracted from the DC output value of this BIT detection circuit without influencing the high frequency performance of the circuit under test. By using this methodology the gain fluctuation of LNA due to PVT variations is also investigated. The achieved results show that this BIT detection circuit can be used to test RF circuits, do functional verification and monitor the performance fluctuation due to the PVT variations without affecting the HF performance of tested RF integrated circuits.

\section{ACKNOWLEDGEMENTS}

This work is supported by Enterprise Ireland under the Commercialization Fund.

\section{REFERENCES}

[1] J.Ferrario, R.Wolf, H.Ding, "Moving from mixed signal to RF test hardware development", IEEE Intl. Test Conference, pp.948 - 956, 2001

[2] Wai Yuen Lau, "Measurement challenges for on-wafer RF-SOC test", 27th Annual IEEE/SEMI International Electronics Manufacturing Technology Symposium, pp.353 - 359, 2002

[3] M.Negreiros, L.Carro, A.A.Susin, "Low cost on-line testing of RF circuits", 10th IEEE International On-Line Testing Symposium, pp.73 - 78, 2004

[4] D.C.Doskocil, "Advanced RF built in test" AUTOTESTCON '92. IEEE Systems Readiness Technology Conference, pp.213 - 217

[5] M.E.Goff, C.A.Barratt, "DC to $40 \mathrm{GHz}$ MMIC power sensor", Gallium Arsenide Integrated Circuit (GaAs IC) Symposium, pp. 105 - 108, 1990

[6] S.S.Akbay, A.Halder, A.Chatterjee, D.Keezer, "Low-cost test of embedded RF/analog/mixed-signal circuits in SOPs", IEEE Transactions on Advanced Packaging, Vol. 27, No. 2, pp.352 - 363, 2004 
[7] A.Chatterjee, A.Keshavarzi, A.Patra,

S.Mukhopadhyay, "Test methodologies in the deep submicron era - analog, mixed-signal and RF", 18th International Conference on VLSI Design, pp.12 - 13, 2005 ,

[8] S.Tanoi, Y.Tokunaga, T.Tanabe, K.Takahashi, A.Okada, M.Itoh, Y.Nagatomo, Y.Ohtsuki, M.Uesugi, "On-wafer BIST of a 200-Gb/s failed-bit search for 1-Gb DRAM", IEEE Journal of Solid-State Circuits, Vol. 32, No. 11, pp.1735 - 1742, 1997,

[9] Jiun-Lang Huang, Chee-Kian Ong, Kwang-Ting Cheng, "A BIST scheme for on-chip ADC and DAC testing", Design, Automation and Test in Europe Conference and Exhibition, pp.216 - 220, 2000

[10] Chujen Lin, L.Haynes, P.Mandava, P.Prasad, "Automatic BIST design tool for mixed-signal circuits", AUTOTESTCON '98. IEEE Systems Readiness Technology Conference, pp.97 - 102, 1998

[11] Wu Zhiqiang, J.Takei, A.Davari, "RF testing of OFDM and CI/OFDM signal over satellite channel", Proceedings of the Thirty-Seventh Southeastern Symposium on System Theory, pp.139 - 143, 2005

[12] R.R.Hatch, R.Brandeberry, W.Knox, "Universal high speed RF/microwave test system", AUTOTESTCON 2003. IEEE Systems Readiness Technology Conference, pp.161 - 171, 2003

[13] A.Valdes-Garcia, R.Venkatasubramanian, R.Srinivasan, J.Silva-Martinez, E.Sanchez-Sinencio, "A CMOS RF RMS detector for built-in testing of wireless transceivers", 23rd IEEE VLSI Test Symposium, pp.249 $-254,2005$

[14] A.Chu, H.M.Cronson, J.F.Devine, S.Soares, M.N.Soloman, H.J.Lezec, C.R.Musil, "RE built-in test and enabling technologies for integrated diagnostics", AUTOTESTCON '92. IEEE Systems Readiness Technology Conference, pp.207 - 211, 1992

[15] S.Sermet Akbay, A.Chatterjee, "Built-in test of RF components using mapped feature extraction sensors", 23rd IEEE VLSI Test Symposium, pp.243 - 248, 2005

[16] S.Bhattacharya, A.Chatterjee, "Use of embedded sensors for built-in-test RF circuits", IEEE Intl. Test Conference, pp.801 - 809, 2004

[17] S.Bhattacharya, A.Chatterjee, "A built-in loopback test methodology for RF transceiver circuits using embedded sensor circuits", 13th Asian Test Symposium, pp. 68 - 73, 2004

[18] T.B. Tarim, M.Ismail, "Robust design of low power CMOS analogue integrated circuits", IEE Proceedings on Circuits, Devices and Systems, Vol. 148, No. 4, pp. 197-204, 2001

[19] M. Nekili, Y. Savaria, G. Bois, "Spatial characterization of process variations via MOS transistor time constants in VLSI and WSI", IEEE Journal of Solid State Circuits, Vol.34, No.1, pp.80-84, 1999

[20] J.-P.Curty, N.Joehl, K F.rummenacher, C.Dehollain,
M.J.Declercq, “A model for /spl mu/-power rectifier analysis and design", IEEE Transactions on Circuits and Systems I: Regular Papers, Vol. 52, No. 12, pp.2771 2779, 2005 